\title{
Jacquard A. et Amblard H., 2014, Réinventons l'humanité, Sang de la terre, Paris, 156 pages
}

José Serrano

\section{(2) OpenEdition}

1 Journals

\section{Édition électronique}

URL : http://journals.openedition.org/developpementdurable/10845

DOI : 10.4000/developpementdurable. 10845

ISSN : 1772-9971

Éditeur

Association DD\&T

\section{Référence électronique}

José Serrano, "Jacquard A. et Amblard H., 2014, Réinventons l'humanité, Sang de la terre, Paris, 156 pages », Développement durable et territoires [En ligne], Vol.6, $n^{\circ} 1$ | Mars 2015, mis en ligne le 24 avril 2015, consulté le 22 septembre 2020. URL : http://journals.openedition.org/developpementdurable/ 10845 ; DOI : https://doi.org/10.4000/developpementdurable.10845

Ce document a été généré automatiquement le 22 septembre 2020.

Développement Durable et Territoires est mis à disposition selon les termes de la licence Creative Commons Attribution - Pas d'Utilisation Commerciale 4.0 International. 


\section{Jacquard A. et Amblard H., 2014, Réinventons l'humanité, Sang de la terre, Paris, 156 pages}

José Serrano

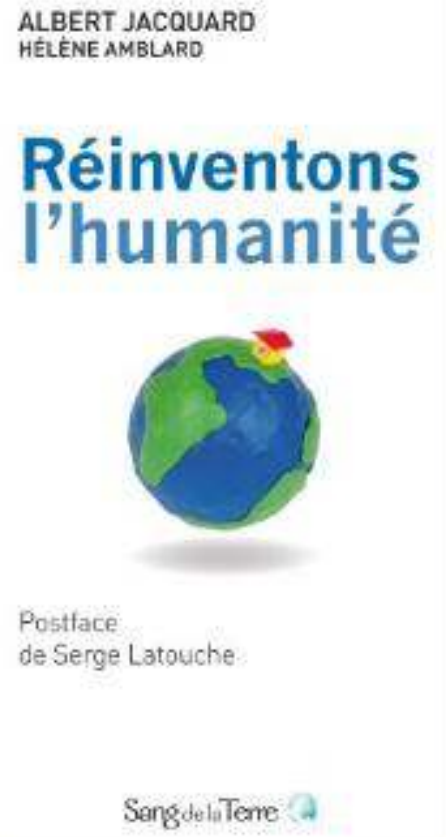

\section{Réinventons} I'humanité

Postface

de Serge Latouche

SangdelaTerve

1 Réinventons l'humanité est un livre posthume d'Albert Jacquard. Il est structuré en deux parties : la première alterne pensées personnelles et réflexions philosophiques d'Albert Jacquard et a été écrit par la journaliste Hélène Amblard, la seconde est une postface assez longue ( $40 \mathrm{p}$.) du professeur d'économie Serge Latouche qui dénonce l'impasse de la croissance comme mode de développement de la société. 
2 L'originalité du texte est la première partie qui alterne réflexions théoriques et témoignages intimes d'Albert Jacquard. Il n'y a pas véritablement de lien direct entre les deux mais leur juxtaposition aboutit à proposer une posture pour une amélioration de notre société.

La première partie du livre développe trois thèmes :

- le rapport à la nature

- la fraternité entre les hommes

- la responsabilité de l'homme par rapport à son devenir.

4 Le rapport à la nature est abordé sur le plan sensible et réflexif. Dans ses pensées personnelles, Albert Jacquard nous livre un rapport contemplatif et sensible. La maison de campagne, l'observation des paysages, mais aussi les parcs urbains sont autant de moments d'émerveillement et d'apaisement. Albert Jacquard propose un accès aisé à la nature pour pouvoir bénéficier de ces moments. Mais il fait remarquer que la société de consommation et le progrès techniques nous éloigne de la nature. C'est pourquoi, les lieux de contacts avec la nature sont à construire et à préserver.

5 Albert Jacquard rejette la société industrielle et capitaliste. Elle mène à la ruine car elle se heurte à la finitude de la planète qui ne peut alimenter de manière croissance et indéfinie le moteur d'une économie fondée sur la consommation. L'appropriation privée des ressources naturelles est aussi un obstacle à la fraternité car elle exclut ceux qui ne possèdent pas. Albert Jacquard propose un gouvernement mondial des ressources naturelles qui sont un bien commun de l'humanité et qui ne devraient pas être exploitées par une minorité. Ces propos sont dispersés dans plusieurs chapitres mais ils défendent l'idée d'une nature accessible à tous et dont les bienfaits sont pour tous.

6 Le contact avec la nature est propice aux retrouvailles intimes ou familiales. Les parcs urbains, mais aussi la maison de campagne sont des lieux privilégiés pour un enracinement. Pour Albert Jacquard, la construction de liens familiaux n'est pas un repli sur soi. Dans son exemple Albert Jacquard montre qu'il appartient à chacun de trouver son coin de nature et d'y installer un nid pour tous les membres de la famille. Celui-ci n'est pas forcément la reproduction de la maison familiale de la génération précédente. Albert Jacquard montre que l'important est de trouver le lieu qui convient et de l'enrichir en y apportant la vie sans être prisonnier de l'héritage.

7 Le vivre ensemble, la fraternité est le second thème développé. Comme pour le thème de la nature, celui-ci est exposé à travers l'exemple personnel et la réflexion philosophique. Le vivre ensemble, les liens de fraternités sont illustrés concrètement par l'exemple de la maison familiale et des relations de voisinage. La réflexion philosophique est une critique de la société de consommation et de la propriété privée. La propriété privée peut être excluante et dominatrice. Les possédants exercent une domination sur ceux qui ne possèdent pas. Albert Jacquard critique la société mercantile. Ce que critique Albert Jacquard ce ne sont pas les échanges en tant que tels mais ceux qui sont stérilisants. Il me semble que l'on n'est pas très loin de l'idée d'une perversion des échanges. La société de consommation est basée sur une majorité de produits inutiles voire nocifs (comme les subprimes par exemple). Pour Albert Jacquard, cette société est révolue et la crise actuelle n'est pas un ajustement mais le symptôme de la transformation de la société vers un autre modèle. Albert Jacquard ne 
dit pas ce que doit être la nouvelle société mais son exemple personnel illustre ce que devrait nous procurer concrètement cette société : contact avec la nature et fraternité.

Nous en venons au troisième thème celui de la responsabilité de l'homme par rapport à son devenir collectif. Albert Jacquard souligne la singularité de l'individu et de l'espèce humaine. Pour le généticien, chaque individu est unique car il est le fruit du hasard, sa combinaison génétique est unique. Il en découle que nul homme n'est supérieur aux autres mais aussi que chaque homme est irremplaçable et donc sacré. L'espèce humaine occupe aussi une place singulière car douée de raison. Les hommes sont responsables de leurs choix envers eux-mêmes et envers leurs descendants. Albert Jacquard pousse à utiliser cette capacité réflexive et cette responsabilité pour remettre en cause le modèle actuel de société. Mais, il se garde bien de donner une solution. Le propos est d'adopter une démarche réflexive pour faire un choix éclairé. La vérité n'existe pas car elle dépend du point de vue adopté. L'important est de réfléchir, toujours réfléchir pour avancer et construire une société meilleure.

Du point de vue du développement durable, l'intérêt principal du livre se trouve ici par rapport au "penser globalement, agir localement». Le développement durable préconise un changement d'échelle pour analyser les enjeux environnementaux et sociétaux. Si ce changement d'échelle permet de saisir les enjeux, la mise en œuvre concrète et locale n'est pas évidente. Cette tension est illustrée ici par l'écart entre les pensées personnelles et les réflexions philosophiques. Cet écart n'est pas résolu par une analyse réflexive mais par une posture. Il ne s'agit pas d'articuler les échelles à travers la compréhension de mécanismes ou de dynamiques environnementales ou socioéconomiques mais d'adopter une position ouverte, fraternelle et réflexive commune pour le quotidien ou pour le collectif. L'accès aisé à la nature vaut pour l'individu à travers son expérience sensible et domestique mais aussi pour le collectif. La fraternité vaut pour l'individu qui tisse des liens de voisinage et familiaux mais aussi pour l'ensemble des hommes, l'humanité. C'est donc aussi en soi que se trouvent les ressources et les réponses aux questions qui se posent à nous. Ce point de vue a le mérite de nous responsabiliser et, peut-être, de nous faire abandonner une posture critique tournée exclusivement vers l'extérieur pour nous faire aussi considérer notre propre changement.

10 La seconde partie du livre est la post face de Serge Latouche. Elle prolonge la critique de la société de consommation. C'est une critique radicale qui prône la décroissance, ce qui va au-delà du développement durable qui lui ne la remet pas explicitement en cause.

11 Les arguments développés sont :

- la finitude de la terre et l'impasse d'une croissance économique de raison géométrique supérieure à un

- l'emballement d'un modèle de société basé sur la croissance car pour soutenir la croissance et avoir une économie dynamique, il faut générer une croissance sans cesse supérieure.

- le mensonge des bienfaits du capitalisme qui concentre les richesses dans les mains de quelques privilégiés en aliénant le plus grand nombre. L'augmentation de la richesse n'a permis d'éradiquer ni la souffrance ni la misère.

12 Serge Latouche dénonce la supercherie de la croissance comme solution pour résorber le chômage ainsi que la myopie voire l'irresponsabilité des dirigeants qui ne renoncent pas à la croissance alors qu'il est simple de comprendre l'impasse de la croissance et la nécessité de l'anticiper longtemps à l'avance. En effet ce qu'Albert Jacquard appelle le 
théorème du nénuphar et qui est repris ici illustre l'effet d'accélération brutal propre aux suites géométriques de raisons supérieures à un. Ces suites progressent peu au début puis de manière vertigineuse.

Ce que préconise Serge Latouche est la décroissance. Le progrès technologique ne permet pas de sortir de l'impasse de la croissance au mieux il ne fait que retarder la crise mais ce sera insuffisant. La substitution du capital naturel par un capital technologique ne permet pas de s'émanciper des limites de la terre. L'installation d'une économie circulaire est insuffisante car les prélèvements excèdent déjà la capacité de renouvellement des ressources naturelles. Il convient de faire baisser le niveau de consommation global.

14 Sege Latouche rassure en rappelant que faire la décroissance n'est pas un cataclysme. Un taux de décroissance géométrique légèrement supérieur à un produit des effets lents au début mais très significatifs sur une cinquantaine d'années.

15 La post face de Serge Latouche vulgarise l'idée de décroissance et donne les références pour un approfondissement. Le texte manque de force de démonstration. La sagesse de l'escargot est proposée comme piste de développement. Sous cette métaphore Serge Latouche préconise : la lenteur, le rassemblement communautaire pour délibérer sur notre destin et une ouverture à soi et aux autres. On reste sur sa faim quant à l'alternative proposée.

\section{AUTEUR}

\section{JOSÉ SERRANO}

Maître de conférences en aménagement de l'espace et urbanisme à l'Université FrançoisRabelais-Tours, José Serrano est rattaché l'UMR Citères 7324. Docteur en agronomie, il est spécialiste des conflits d'usages dans les espaces périurbains. Il s'intéresse particulièrement aux questions de multifonctionnalité des espaces. 